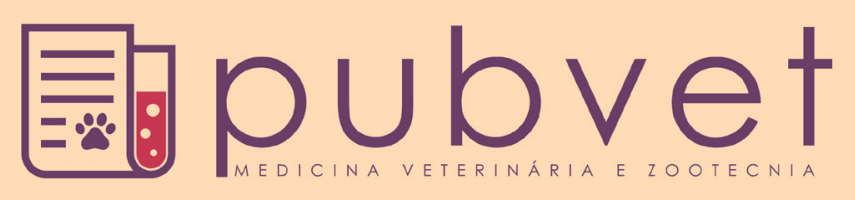

HTTP://DX.DOI.ORG/10.22256/PUBVET.V11N7.680-688

\title{
Deslocamento de abomaso à esquerda: Revisão
}

\author{
Yanne Aciole da Silva ${ }^{1}$, Wendel de Souza Mendonça ${ }^{1}$, Alcir Martins Pereira ${ }^{1}$, Francisco \\ das Chagas Cardoso Junior ${ }^{1}$, Francisco Solano Feitosa Junior ${ }^{2}$, Taciana Galba da Silva \\ Tenório $^{2^{*}}$
}

${ }^{1}$ Médico(a) Veterinário(a) Autônomo.

${ }^{2}$ Professor(a) do Departamento de Clínica e Cirurgia Veterinária da Universidade Federal do Piaui, UFPI, Teresina, PI.

*Autor para correspondência, E-mail: tacianagalba@yahoo.com.br

RESUMO. O deslocamento de abomaso corresponde à patologia responsável pela maioria das intervenções cirurgias no abdome em vacas leiteiras, no entanto, pode afetar bovinos de qualquer idade e sexo. O deslocamento pode dar-se à esquerda (DAE) ou à direita (DAD) com ou sem torção abomasal (VA). Sendo o DAE mais frequente e relacionado ao periparto, onde o abomaso encontra-se deslocado de sua posição a anatômica para o lado esquerdo, em função de diversos fatores que levam a hipomotilidade ou atonia do órgão e, consequentemente o acúmulo de gás. Fatores predisponentes para ocorrência de DAE são: balanço energético negativo, alimentação pobre em fibras e rica em concentrados, manejo na alimentação de transição, idade, sexo, genética (raça, profundidade corporal), condições climatéricas, doenças concomitantes. $\mathrm{O}$ tratamento consiste no reposicionamento do órgão em seu local fisiológico, e a do mesmo por técnicas cirúrgicas abertas ou fechadas. A reposição de eletrolíticos é importante para a recuperação do animal, bem como, o restabelecimento da motilidade abomasal. Quando não ocorrem complicações, os índices de retorno à produção são elevados entre os casos de DAE O objetivo desse trabalho é uma discussão a respeito da clínica completa do DAE.

Palavras chaves: Abomaso, bovino, clínica

\section{Abomaso Shift left: Review}

ABSTRACT. The displaced abomasum corresponding to the pathology responsible for the majority of surgical operations on the abdomen in dairy cows, however, affect animals of all ages and sex. The displacement may be give to the left (LDA) or right (DAD) with or without twist abomasal (VA). It is the most common AEDs and related peripartum where the abomasum is displaced from its anatomic position to the left side, depending on various factors that lead to hypomotility or atony of the body and consequently the gas accumulation. predisposing factors for the occurrence of AEDs are: negative energy balance, diet low in fiber and high in concentrates, handling the transition of power, age, gender, genetics (breed, body depth), weather conditions, concomitant diseases. The treatment consists in the adjustment of the body in their physiological location, and the same or closed by open surgical techniques. The electrolyte replacement is important to the recovery of the animal, as well as the restoration of abomasal motility. When no complications occur, the rates of return to production are high among the cases of DAE. The aim of this work is an increasing discussion about the complete clinical AED.

Keywords: abomasum, cattle, clinical 


\section{Desplazamiento de abomaso a la izquierda: Revisión}

RESUMEN. El desplazamiento de abomaso corresponde a la patología responsable por la mayoría de las intervenciones quirúrgicas abdominales en vacas lecheras, sin embargo, puede afectar bovinos de cualquier edad y sexo. El desplazamiento puede darse a la izquierda (DAE) o a la derecha (DAD) con o sin torsión abomasal (VA). Siendo el DAE más frecuente y relacionado al periparto, donde el abomaso se encuentra desplazado de su posición a la anatómica hacia el lado izquierdo, en función de diversos factores que llevan a la hipomotilidad o atonía del órgano y, consecuentemente la acumulación de gas. Los factores predisponentes para la ocurrencia de DAE son: balance energético negativo, alimentación pobre en fibra y rica en concentrados, manejo en la alimentación de transición, edad, sexo, genética (raza, profundidad corporal), condiciones climatéricas, enfermedades concomitantes. El tratamiento consiste en el reposicionamiento del órgano en su sitio fisiológico por medo de técnicas quirúrgicas abiertas o cerradas. La reposición de electrolíticos es importante para la recuperación del animal, así como el restablecimiento de la motilidad abomasal. Cuando no ocurren complicaciones, los índices de retorno a la producción son elevados entre los casos de DAE. El objetivo de este trabajo es una discuitir al respecto de la clínica completa del DAE.

Palabras claves: abomaso, bovino, clínica

\section{Introdução}

A posição do abomaso pode varia com o estado gestacional, a postura do animal e o volume e dinâmica ruminal, anatomicamente encontra-se no plano medial entre o saco ventral do rúmen e o omaso, situado levemente à esquerda (Gordo, 2009). Quando deslocado entre o rúmen e a parede abdominal esquerda constitui o DAE, e ao deslocar-se completamente para o lado direito da cavidade abdominal ocorre deslocamento de abomaso á direita (DAD), condição de risco para desenvolvimento do vólvulo abomasal (VA) (Riet-Correa et al., 2007). O deslocamento de abomaso é responsável pela maioria das cirurgias abdominais em vacas leiteiras, sendo 85 a $95 \%$ dos casos atribuído ao deslocamento de abomaso à esquerda (DAE) (Radostits et al., 2010). Acomete $1,8 \%$ do rebanho brasileiro em período pós-parto (Corassin, 2004). Os primeiros relatos de deslocamento de abomaso datam de 1898 descrito por Carougeau e Prestat em vitelo com VA, publicado no "Journal de Médecine Vétérinaire et de Zootechnie" da Escola de Medicina Veterinária de Lyon em França. Bregg e Ford relataram os primeiros casos de DAE em animais adultos no ano de 1950 (Breukink, 1992).

A importância econômica está associada à produção de leite e custos com tratamentos, bem como, aumento do intervalo entre parto, perda de peso e descarte ou mortalidade de matrizes de alto valor produtivo (Geishauser et al., 2000).

Diante do exposto o objetivo desta revisão é abordar aspectos relacionados à etiologia, fatores de risco, epidemiologia, patogenia, sinais clínicos, diagnósticos, prognósticos, tratamentos, prevenção e controle.

\section{Etiologia}

De acordo com Dirksen et al. (1993), a dilatação do abomaso é antecedida por transtorno de motilidade abomasal (hipomotilidade ou atonia). Contudo, a etiologia do deslocamento de abomaso a esquerda é considerada multifatorial, e frequentemente, está relacionada ao princípio com a concentração elevada de carboidratos altamente fermentáveis em relação à quantidade de fibra efetiva da dieta, principalmente no período de transição, que vai desde as duas semanas pré-parto até as duas a quatro semanas pós-parto (Coppock et al., 1972, Cameron et al., 1998, Shaver, 1997, Doll et al., 2009, Gordo, 2009, Câmara et al., 2009).

\section{Fatores de risco}

Pesquisadores ressaltam a importante associação entre o balanço energético negativo no pré-parto refletindo um aumento na concentração de ácidos graxos não esterificados e o deslocamento de abomaso para esquerda (Cameron et al., 1998, Van Winden et al., 2004). Portanto, dentro os principais fatores de risco relacionados ao manejo nutricional no período pré-parto de vacas e consequentemente ao balanço energético negativo: elevado níveis de condição corporal, inadequado fornecimento de dieta, dietas altamente energéticas e estações de inverno e 
verão, alto mérito genético e baixa parição (Radostits et al., 2010). A diminuição da ingestão de matéria seca (aproximadamente 35\%) na última semana pré-parto, e o lento aumento no pós-parto aumenta as concentrações de triglicérides hepáticos (Bertics et al., 1992). Deste modo, dietas com níveis superiores a 1,65 Mcal de energia líquida $/ \mathrm{kg}$ de matéria seca em período seco induzem a obesidade procedendo diminuição no consumo de matéria seca no momento do parto. Assim como, durante os meses quentes de verão também interfere na ingestão de matéria seca por esses animais, sugerindo que a lipidose hepática, comum em vacas com elevada condição corporal, é considerada um fator de risco para o DAE (Cameron et al., 1998, Radostits et al., 2010, Gordo, 2009).

Alimentação rica em concentrado aumenta a passagem da ingesta ruminal, induzindo ao aumento nos níveis de ácidos graxos voláteis, que por sua vez pode inibir a motilidade abomasal (Van Winden (Van Winden et al., 2004, Divers and Peek, 2007). Consequentemente o fluxo da ingesta do abomaso para o duodeno fica impossibilitado, acumulando-se no abomaso. De modo que, o acúmulo elevado de metano e dióxido de carbono nesse órgão, acarreta em sua distensão e deslocamento (Radostits et al., 2010). Contudo, a importância do aumento da concentração de AGV no abomaso como causa de atonia neste órgão é ainda controversa (Radostits et al., 2010). Deve se levar em consideração que dietas pobres em energias fornecidas no final do pré-parto também é um fator de risco para ocorrência de DAE, devido à falha no aumento da capacidade de absorção das papilas ruminais e ineficiência na adaptação da microflora ruminal à ingestão de dietas do pós-parto, com níveis energéticos superiores. Assim, incidência de lipidose hepática e Cetose podem ser reduzidas pelo esse aumento da absorção de energia (Gordo, 2009). Dietas de vaca leiteiras contendo menos de $16-17 \%$ de fibra bruta predispõem ao deslocamento de abomaso. Além disso, um aumento de $17 \%$ na incidência de DAE foi observado no fornecimento de uma dieta experimental completamente peletizada, em comparação ao grupo de vacas que receberam feno de alfafa solta, silagem de sorgo e $18 \%$ concentrado de Proteína bruta, na qual, a incidência foi de 1,6\% (Dawson et al., 1992). O aumento dos ácidos graxos e a produção de gás consequente do acumulo ruminal e aumento da fermentação decorrente de ingestão de ração peletizada finamente moída e a fibra de comprimento curto podem ser fatores de risco para o DAE, por provocarem reduzida função ruminal e supressão do apetite (Radostits et al., 2010, Sexton et al., 2007).

A cetose clínica diagnosticada antes DAE, associada a uma baixa ingestão de matéria seca, é um fator de risco relevante. A cetose origina da diminuição do enchimento ruminal e consequentemente em seu volume, reduzindo a motilidade dos pré-estômagos e do abomaso (Radostits et al., 2010).

As raças leiteiras são mais predisponentes ao deslocamento de abomaso, visto que, a seleção intensiva de bovinos com uma alta estatura e grande profundidade corporal aumenta o risco de ocorrência da enfermidade (Divers and Peek, 2007, Panelli, 2014), uma vez que, a distância vertical mais elevada entre o abomaso e o duodeno descendente dificultará o esvaziamento do abomaso (Câmara et al., 2009, Panelli, 2014). Todavia, em vários estudos não há resultados significativos com relação à alta produção leiteira ou o alto potencial de produção e o DAE (Zwald et al., 2004, Radostits et al., 2010). Pesquisas indicam aumento proporcional da ocorrência de deslocamento de abomaso com a idade, evidenciando maior fator de risco entre quatro e sete anos de idade (Radostits et al., 2010). No entanto, é relevante ressaltar que bovinos em qualquer idade estão propensos a essa patologia (Geishauser et al., 2000, Divers and Peek, 2007).

Acredita-se que o parto aparenta ser a condição precipitante mais frequente do deslocamento abomasal. Pois, no estágio avançado de prenhez, o volume uterino aumentado toma uma parte do espaço do rúmen, este é levantado do piso abdominal pelo útero, e o abomaso é deslocado anteriormente e para a esquerda, sob o rúmen. Logo após o parto, com a saída do terneiro e fluídos existe uma propensão anatômica para o abomaso luxar-se, porque aumenta o espaço abdominal quando o rúmen moderadamente vazio continua a encurralar o abomaso, sobretudo se este está atónico ou distendido com alimento, especialmente caso a vaca foi submetida a uma dieta com excesso de concentrado (Radostits et al., 2010).

Existem determinadas doenças concomitantes que induz ao deslocamento de abomaso que causam anorexia e inapetência, procedendo na diminuição do volume ruminal. As enfermidades comumente associadas ao deslocamento de abomaso são vinculadas por processos febris e 
inflamatórios, tais como: retenção placentária, metrite, mastite severa, úlcera abomasal, cetose, fígado gordo, hipocalcémia, indigestão (Gordo, 2009, Radostits et al., 2010).

A febre, endotoxinas e mediadores infamatórios induzem a supressão das contrações retículo-rúmen, pelo efeito a depressor direto do núcleo central vagal e a estimulação do tônus da musculatura lisa que ocasiona à inibição dos reflexos. Todavia, não há relato sobre esses efeitos no abomaso, todavia, há estudo das mesmas consequências inibitórias em outras espécies, sobre as funções gastrointestinais motoras e secretoras (Gingerich and Murdick, 1975, McGuirk and Butler, 1980, Gordo, 2009).

\section{Epidemiologia}

A prevalência do deslocamento de abomaso varia de rebanho para rebanho dependendo da localização geográfica, práticas de manejo, clima, entre outros fatores (Câmara et al., 2009). Ocorre principalmente, em vacas leiteiras de grande porte recém-paridas, mantidas estabuladas e alimentação rica em grãos, assim como também em vacas criadas a pasto (Radostits et al., 2010).

O DAE representa a causa mais comum para cirurgia abdominal nos bovinos leiteiros nas primeiras seis semanas pós-parto (Radostits et al., 2010). Todavia, pode também acometer outras categorias como bezerros, touros, novilhas (Dirksen et al., 1993, Riet-Correa et al., 2007) e, em vacas não gestantes a frequência é de menos de $10 \%$ dos casos, enquanto que em bovinos de corte há poucos relatos de deslocamento de abomaso (Roussel et al., 2000).

\section{Patogenia}

O abomaso hipomotílico e cheio de gás desloca-se ventralmente e sob o rúmen, através da parede abdominal esquerda, lateralmente ao baço e ao saco dorsal do rúmen. O deslocamento do piloro e duodeno é subsequente à movimentação do fundo e a grande curvatura do abomaso. Por sua vez, ocorre o deslocamento do omaso, retículo e fígado. Sempre ocorre ruptura zona de fixação do omento maior ao abomaso decorrente do deslocamento do abomaso (Radostits et al., 2010).

Eventualmente, o segmento deslocado do abomaso detém-se preso entre o retículo e o diafragma, ocasionado o "deslocamento do abomaso anterior", responsável por $12 \%$ dos casos em um estudo com 161 deslocamentos de abomaso (Zadnik, 2003, Radostits et al., 2010).
Esse tipo de deslocamento resulta em inanição severa e comprometimento na digestão e movimento da ingesta. A atonia abomasal e secreção constante de ácido hipoclorídrico provocam alcalose metabólica moderada com hipocloremia e hipocalemia, bem como, alteração no influxo da ingesta para o duodeno e úlceras do abomaso em casos crônicos.

Quando ocorre a rotação de todos os estômagos acarreta alterações na função esofágica, dificulta a passagem do bolo alimentar. Contudo, a fluxo de líquido na porção obstruída não é completamente inibida, possibilitando certo esvaziamento de fluídos, de modo consequente, o deslocamento dificilmente é gravíssimo. (RP). Todavia, Gordo (2009) descreve que a motilidade abomasal é coordenado pela tonicidade do nervo vago, e dependente das informações do sistema autônomo. Por sua vez, uma fração dessas informações está relacionada com as concentrações de glucose e insulina plasmáticas. Assim, elevação dos níveis dessas substâncias, induzido pelo aumento da concentração sanguínea de ácidos gordos de cadeia curta (butirato, valeriato), stress, hormonas de crescimento, infecção e endotoxinas (Van Meirhaeghe et al., 1988, DeCupere et al., 1992) intensificam a tonicidade vagal e, conseguinte aumenta a motilidade e secreção gástrica.

\section{Sinais clínicos}

O curso DAE é variável e geralmente os animais acometidos manifestam anorexia total ou moderada, consequentemente redução gradativo da produção de leite, diminuição da taxa de ruminação e do consumo de concentrado, preservando o interesse pela forragem (Divers and Peek, 2007, Radostits et al., 2010, Panelli, 2014), fezes escassas podendo serem diarreicas. Ainda apresenta enoftalmia, refletindo certo estado de desidratação, Cetose em graus variáveis, cifose evidenciando sinais de dor, e depressão (Radostits et al., 2010, Gordo, 2009, Panelli, 2014). Os parâmetros fisiológicos temperatura e frequências respiratória e cardíaca mantêm se dentro da normalidade, porém ocasionalmente, pode ocorrer uma arritmia cardíaca pela alcalose metabólica (Gordo, 2009, Radostits et al., 2010, Panelli, 2014).

$\mathrm{Na}$ inspeção abdominal, observa-se concavidade na fossa paralombar esquerda, proporcionada pela redução do volume ruminal e posicionamento deste medialmente (Radostits et 
al., 2010). Em DAE intenso, onde o abomaso encontrasse tenso e com aumento de volume timpânico, ou mesmo, em casos que o animal encontra-se magro é possível observar leve arqueamento das costelas por pressão do abomaso e a curvatura do abomaso pode ser palpável na porção cranial da fossa paralombar (Dirksen et al., 1993, Divers and Peek, 2007).

A dinâmica ruminal apresenta movimentos de baixa amplitude e frequência reduzida, evidencia motilidade desse órgão, porém, ocasionalmente a auscultação pode ser inaudível, também sugestível de DAE. Sons metálicos abomasais são observados em uma área delimitada por uma linha imaginária partida do centro da fossa paralombar esquerda até a tuberosidade do rádio, repetindo-se varia vezes por minutos ou com intervalos longos, de até 5 minutos entre eles, sem nenhuma relação com os sons ruminais. Ao balotamento ou sucussão no abdômen ventral esquerdo, é notável auscultação de sons de líquidos e de "chapinhar" (Radostits et al., 2010, Panelli, 2014).

Uma ressonância metálica "ping" captada pela percussão simultânea à auscultação entre a $9^{\mathrm{a}} \mathrm{e}$ a $13^{\mathrm{a}}$ costelas, no terço superior da parede abdominal esquerda é patognomônico do DAE (Divers and Peek, 2007, Radostits et al., 2010).

Todavia, conforme o abomaso se posiciona e a quantidade de gás acumulado determina o tamanho dessa área de ressonância metálica, que por sua vez pode oscilar em intervalos curtos de tempo e locais de percepção em um mesmo animal. Esta situação caracteriza o "Deslocamento Abomasal flutuante". Ao exame de palpação retal, aparenta esvaziamento da porção superior direita do abdômen, em razão do rumem encontra-se com o volume menor que o esperado. No entanto, pode notar por ventura, um timpanismo ruminal crônico (Gordo, 2009, Radostits et al., 2010, Panelli, 2014).

\section{Diagnóstico clínico}

O diagnóstico do DAE é baseado no histórico e sinais clínicos que o animal apresenta. Østergaard and Gröhn (2000) vacas no pós-parto que diminuíram o consumo de mais de $2 \mathrm{~kg}$ de matéria seca/dia são relevantes para DAE. A confirmação pode ser obtida através da auscultação e percussão terço ventral a partir do $8^{\circ}$ espaço intercostal até a fossa paralombar do lado esquerdo do paciente, detectando-se o som metálico característico de ping (Radostits et al., 2010). O exame retal auxilia no diagnóstico, quando se tem a percepção de vazio no quadrante dorsal direito, e ao palpar o abomaso do lado esquerdo do saco cego caudodorsal do rúmen, sugerindo deslocamento das vísceras (Radostits et al., 2010).

\section{Exames complementares}

Animais acometidos com DAE não apresentam alterações significativas no hemograma, porém podem expor valores elevados para hemoglobina e hematócrito decorrente a hemoconcentração em casos graves (Cameron et al., 1998, Cardoso et al., 2008, Radostits et al., 2010) ou consequente à anorexia (Cardoso et al., 2008). Alguns casos, o animal pode indicar leucocitose por neutrofilia, linfopenia ou mesmo, por eosinopenia. Essas condições são peculiares em animais estressados, elevando os níveis plasmáticos de cortisol ou adrenalina (Jones and Allison, 2007).

$\mathrm{Na}$ bioquímica sérica, expressa alcalose metabólica com hipocalemia e hipocloremia resultante da atonia abomasal que dificulta o fluxo do ácido clorídrico para o duodeno. Esses teores também encontram reduzidos quando o animal possui lipidose hepática (Svendsen, 1969, Smith, 2006, Divers and Peek, 2007). Além disso, quando existe perda de água hipotônica ou excreção renal de sódio, para compensar a alcalose, ocorre hiponatremia (Rohn et al., 2004). Ainda é demonstrado aumento dos níveis de AGNE e de AST (Gordo, 2009). Grünberg et al. (2005) constatam valores reduzidos de fósforo em animais com deslocamento de abomaso, consequente da redução da alimentação e de lesões hepáticas. Enquanto, Delgado-Lecaroz et al. (2000) relatam leve queda nos níveis de magnésio plasmático em animais com DAE.

A pesquisa de corpos cetônicos, indicando cetose, pode ser realizada na urina, no sangue e no leite, porém, neste último os resultados são usados como métodos presuntivos realizado na segunda semana após o parto (Geishauser et al., 1997, Carrier et al., 2004). Cetoses intensas são acompanhadas por alterações no $\mathrm{pH}$ sanguíneo, aumento do aníon gap2 e diminuição nos valores de bicarbonato (Divers and Peek, 2007). Gordo (2009) ressalta que, em vacas com DAE os valores para glicose e insulina encontram alterados independentes da cetose, apresentando hiperglicemia e hiper-insulinemia.

No exame do líquido peritoneal para diagnóstico de DAE, é evidenciado aumento significativo de haptoglobina sérica, 
malondialdeído, adenosina deaminase, como também observado no exame de sangue, além do aumento na concentração de proteína, ao mesmo tempo, diminuição dos níveis de óxido nitrito (Maden et al., 2012).

A ultrassonografia pode ser utilizada como método eficiente para diagnóstico de DAE. Dessa forma, é realizada entre no $10^{\circ}$ e $12^{\circ}$ espaços intercostais esquerdos no sentido ventro-dorsal, na qual, revela o abomaso em lugar do rúmen, com ingesta líquida na parte ventral e gás na dorsal, e suas pregas entre a ingesta (Gordo, 2009, $\underline{\text { Radostits et al., 2010, }}$ Panelli, 2014).

\section{Diagnóstico diferencial}

Doenças que causam anorexia, cetose, hipomotilidade ou atonia dos pré-estômagos, sons anormais na percussão e auscultação do abdome esquerdo como timpanismo ruminal, indigestão vagal, indigestão simples, cetose primária, reticuloperitonite traumática, actinobacilose da goteira esofágica, peritonite localizada, abcesso sub-peritoneal, carcinoma do cárdia, do abomaso, privação alimentar, síndrome da vaca gorda, colapso ruminal, síndrome do rúmen vazio, bem como, pneumoperitoneu, fisómetra e dilatação e/ou deslocamento do ceco à esquerda são enfermidades para diagnóstico diferencial de DAE (Radostits et al., 2010).

Existe diferença entre o $\mathrm{pH}$ ruminal ( $\mathrm{pH}$ 6-7) e do abomaso (pH 2-3), na aspiração percutânea do gás ou do líquido, portanto, consiste em um meio válido para confirmação da origem do som metálico ping. Enquanto o quadro de cetose pós parto, deve se levar em conta a recidiva em poucos dias após o tratamento, caracterizando a cetose secundária a DAE (Radostits et al., 2010).

\section{Tratamento}

A cura do DAE obtém se com o reposicionamento anatômico do abomaso e o tratamento de suas consequências e doenças concomitantes (Divers and Peek, 2007). É indispensável o tratamento das doenças concomitante e associadas ao DAE. Sugere-se o uso de laxantes orais, ruminatórios, antiácidos ou drogas colinérgicas como conduta terapêutica para restauração da motilidade gastrointestinal. Ao mesmo tempo, para a correção de hipocalcemia, deve se fazer uso soluções de cálcio por via intravenosa lenta ou subcutânea (Divers and Peek, 2007), ainda citam a administração de cloreto de potássio por via oral, na dose de 30 a $120 \mathrm{~g}$ com a finalidade restaurar o equilíbrio eletrolítico.

A técnica de rolamento é empregada para correção do DAE, porém, possui uma alta recidiva, cerca de $50 \%$ dos casos, e pode ocasionar complicações ao animal, como DAD ou volvo abomasal. Nessa técnica o animal é derrubado e posicionado em decúbito dorsal por dois a cinco minutos, durante esse tempo o animal é balançado de um lado para o outro e massageado na região em que o abomaso se encontra, fundamentado na flutuação do abomaso repleto de gás para sua posição fisiológica e esvaziamento de parte do gás, posteriormente o animal é colocado em decúbito esquerdo e imediatamente estimulado a levantar. É realizado a monitorização do abomaso através da auscultação e percussão durante esse procedimento. E ao final, deve se oferecer forragem (Divers and Peek, 2007, Gordo, 2009).

Jang et al. (2003) relacionam a técnica de rolamento e a acupuntura como alternativas eficientes, segura e econômica à cirurgia para solução de casos de DA. Contudo, existem varias técnicas cirúrgicas para a correção do DAE, entre ela a omentopexia paralombar direita (ou método de Hannover), abomasopexia paramedial direita e a abomasopexia paralombar esquerda são as mais empregadas.

A omeontrexia consiste na fixação indireta do abomaso ao flanco direito, através da retração dorso-caudal do omento maior pela incisão abdominal, essa técnica é executada com o animal em estação e foi descrita por Dirksen em 1961. A omentoplexia pode ser combinada a piloroplexia, onde, o piloro juntamente com o omento maior caudal a ele é fixado ao peritônio e músculo transverso do abdômen.

As técnicas de cirurgias fechadas exclusivas para DAE são indicadas por serem mais rápidas e mais baratas (Radostits et al., 2010). No entanto, não tem o reconhecimento do local da sutura possibilitando a ocorrência complicações como peritonites, celulites, deslocamento abomasal ou evisceração, completa obstrução do pré-estômago e tromboflebite da veia subcutânea abdominal, assim como, fixação de outras estruturas como rúmen, intestino ou fixar o abomaso em uma posição equivocada (Radostits et al., 2010). As suturas fechadas para a abomasopexia são feitas através da parede abdominal com agulhas em "C". Nesse método o animal é acomodado em decúbito dorsal e realizado a identificação do abomaso pela 
auscultação e percussão (Bartlett et al., 1995, Gordo, 2009).

\section{Prognóstico}

O prognóstico pode ser bom, 86 a $95 \%$ dos DAE (Dirksen et al., 1993) quando não ocorre complicações, e desfavorável nos casos de ruptura de úlcera do abomaso (Radostits et al., 2010) e $75 \%$ dos casos são considerados prognósticos reservados (Santarosa, 2010).

\section{Controle e prevenção}

Identificar os fatores que predispões ao desenvolvimento do deslocamento é relevante pra a perversão do DAE, bem como tratamentos de enfermidades pós-parto como metrite, mastite e retenção de placenta. As medidas baseiam-se no manejo reprodutivo e manipulação nutricional dos animais susceptíveis. A redução do intervalo entre parto associado ao fornecimento de concentrado durante a fase de gestação e lactação resulta numa melhoria do balanço energético, consequentemente no estado metabólico das vacas na lactação subsequente, e assegurar a ingestão de MS no pós-parto imediato (Panelli, 2014) diminuiu a ocorrência de acidose ruminal e deslocamento de abomaso. Østergaard and Gröhn (2000) defendem que, a adoção da dieta completa (TMR) na fase de lactação reduz o risco de desenvolvimento de DAE.

Monitorização da qualidade do leite em vacas no início da lactação pode ser utilizado como medida de prevenção de DA. De acordo com Mecitoglu et al. (2012) a razão proteína/gordura inferior a 0,63 e o teor de gordura e maior que 5,29 são indicativos de deslocamento de abomaso à esquerda.

\section{Referências Bibliográficas}

Bartlett, P. C., Kopcha, M., Coe, P. H., Ames, N. K., Ruegg, P. L. \& Erskine, R. J. 1995. Economic comparison of the pyloroomentopexy vs the roll-and-toggle procedure for treatment of left displacement of the abomasum in dairy cattle. Journal of the American Veterinary Medical Association, 206, 1156-1162.

Bertics, S. J., Grummer, R. R., Cadorniga-Valino, C. \& Stoddard, E. E. 1992. Effect of prepartum dry matter intake on liver triglyceride concentration and early lactation. Journal of Dairy Science, 75, 1914-1922.
Breukink, H. J. 1992. Abomasal displacement, etiology, pathogenesis, treatment and prevention. Bovine Practitioner, 26, 148-148.

Câmara, A. C. L., Ximenes, F. H. B., Moscardini, A. R. C., Castro, M. B., Godoy, R. F. \& Borges, J. R. J. 2009. Vólvulo abomasal em dois bezerros. Veterinária e Zootecnia, 16, 459464.

Cameron, R. E. B., Dyk, P. B., Herdt, T. H., Kaneene, J. B., Miller, R., Bucholtz, H. F., Liesman, J. S., Vandehaar, M. J. \& Emery, R. S. 1998. Dry cow diet, management, and energy balance as risk factors for displaced abomasum in high producing dairy herds. Journal of Dairy Science, 81, 132-139.

Cardoso, F. C., Esteves, V. S., Oliveira, S. T., Lasta, C. S., Valle, S. F., Campos, R. \& González, F. H. D. 2008. Hematological, biochemical and ruminant parameters for diagnosis of left displacement of the abomasum in dairy cows from Southern Brazil. Pesquisa Agropecuária Brasileira, 43, 141147.

Carrier, J., Stewart, S., Godden, S., Fetrow, J. \& Rapnicki, P. 2004. Evaluation and use of three cowside tests for detection of subclinical ketosis in early postpartum cows. Journal of Dairy Science, 87, 3725-3735.

Coppock, C. E., Noller, C. H., Wolfe, S. A., Callahan, C. J. \& Baker, J. S. 1972. Effect of forage-concentrate ratio in complete feeds fed ad Libitum on feed intake prepartum and the occurrence of abomasal displacement in dairy cows. Journal of Dairy Science, 55, 783-789.

Corassin, C. H. 2004. Determinação e avaliação de fatores que afetam a produtividade de vacas leiteiras: aspectos sanitários e reprodutivos. Universidade de São Paulo, Piracicaba.

Dawson, L. J., Aalseth, E. P., Rice, L. E. \& Adams, G. D. 1992. Influence of fiber form in a complete mixed ration on incidence of left displaced abomasum in postpartum dairy cows. Journal of the American Veterinary Medical Association, 200, 1989-1992.

DeCupere, F., Muylle, E., Van Den Hende, C. \& Oyaert, W. 1992. Metabolic profile tests in high yielding normal cows and in cows suffering from abomasal displacement. Bovine Practitioner, 26, 129-129.

Delgado-Lecaroz, R., Warnick, L. D., Guard, C. L., Smith, M. C. \& Barry, D. A. 2000. Crosssectional study of the association of abomasal displacement or volvulus with serum 
electrolyte and mineral concentrations in dairy cows. The Canadian Veterinary Journal, 41, 301-305.

Dirksen, G., Gründer, H. D. \& Stöber, M. 1993. Exame Clínico dos Bovinos. Guanabara Koogan, Rio de Janeiro.

Divers, T. J. \& Peek, S. 2007. Rebhun's diseases of dairy cattle. Elsevier Health Sciences.

Doll, K., Sickinger, M. \& Seeger, T. 2009. New aspects in the pathogenesis of abomasal displacement. The Veterinary Journal, 181, 90-96.

Geishauser, T., Leslie, K. \& Duffield, T. 2000. Metabolic aspects in the etiology of displaced abomasum. Veterinary Clinics of North America: Food Animal Practice, 16, 255-265.

Geishauser, T., Leslie, K., Duffield, T. \& Edge, V. 1997. An evaluation of milk ketone tests for the prediction of left displaced abomasum in dairy cows. Journal of Dairy Science, 80, 31883192.

Gingerich, D. A. \& Murdick, P. W. 1975. Paradoxic aciduria in bovine metabolic alkalosis. Journal of the American Veterinary Medical Association, 166, 227-230.

Gordo, R. I. N. 2009. Contribuição para o estudo do deslocamento do abomaso numa exploração leiteira da região de Montemor-o-Velho. Faculdade de Medicina Veterinária. Universidade Técnica de Lisboa, Lisboa.

Grünberg, W., Constable, P., Schröder, U., Staufenbiel, R., Morin, D. \& Rohn, M. 2005. Phosphorus homeostasis in dairy cows with abomasal displacement or abomasal volvulus. Journal of Veterinary Internal Medicine, 19, 894-898.

Jang, K. H., Kang, W. M. \& Lee, J. M. 2003. Electroacupuncture and moxibustion for correction of abomasal displacement in dairy cattle. Journal of Veterinary Science, 4, 93-95.

Jones, M. L. \& Allison, R. W. 2007. Evaluation of the ruminant complete blood cell count. Veterinary Clinics of North America: Food Animal Practice, 23, 377-402.

Maden, M., Ozturk, A. S., Bulbul, A. \& Avci, G. E. 2012. Acute-phase proteins, oxidative stress and enzyme activities of blood serum and peritoneal fluid in cattle with abomasal displacement. Journal of Veterinary Internal Medicine, 26, 1470-1475.

McGuirk, S. M. \& Butler, D. G. 1980. Metabolic alkalosis with paradoxic aciduria in cattle.
Journal of the American Veterinary Medical Association, 177, 551-554.

Mecitoglu, Z., Demir, G., Senturk, S., Uzabaci, E. \& Darici, R. 2012. Milk protein/fat ratio on day 7 postpartum as a predictor of left displacement of abomasum. Veterinary Record, 171, 197197.

Østergaard, S. \& Gröhn, Y. T. 2000. Concentrate feeding, dry-matter intake, and metabolic disorders in Danish dairy cows. Livestock Production Science, 65, 107-118.

Panelli, E. M. 2014. Deslocamento do abomaso à esquerda em bovinos leiteiros: da etiologia ao diagnóstico. Faculdade de Medicina $e$ Veterinária. Universidade Estadual de São Paulo, Araçatuba.

Radostits, O. M., Gay, C. C., Blood, D. C., Hinchcliff, K. W. \& McKenzie, R. A. 2010. Clínica Veterinária: um tratado de doenças dos bovinos, ovinos, suínos, caprinos $e$ eqüinos. Guanabara Koogan, Rio de Janeiro.

Riet-Correa, F., Méndez, F., Carmen Schild, M., Riet-Correa, A. L. F., Schild, A. L. \& Carmen, M. M. 2007. Doenças de ruminantes e eqüinos. Varela, Sãp Paulo.

Rohn, M., Tenhagen, B. A. \& Hofmann, W. 2004. Survival of dairy cows after surgery to correct abomasal displacement: 2. Association of clinical and laboratory parameters with survival in cows with left abomasal displacement. Journal of Veterinary Medicine Series A, 51, 300-305.

Roussel, A. J., Cohen, N. D. \& Hooper, R. N. 2000. Abomasal displacement and volvulus in beef cattle: 19 cases (1988-1998). Journal American Veterinary Medical Association, 216, $730-733$.

Santarosa, B. P. 2010. Deslocamento de abomaso em vacas leiteiras. Faculdade de Medicina Veterinária e Zootecnia. Universidade do Estado de São Paulo, Botucatu.

Sexton, M. F., Buckley, W. \& Ryan, E. 2007. A study of 54 cases of left displacement of the abomasum: February to July 2005. Irish Veterinary Journal, 60, 605.

Shaver, R. D. 1997. Nutritional Risk Factors in the Etiology of Left Displaced Abomasum in Dairy Cows: A Review1. Journal of Dairy Science, 80, 2449-2453.

Smith, M. O. 2006. Tratado de medicina interna de grandes animais. Manole, São Paulo. 
Svendsen, P. E. 1969. Etiology and pathogenesis of abomasal displacement in cattle. Nordisc Veterinary Medical, 21, 1-60.

Van Meirhaeghe, H., Deprez, P., Hende, C. \& Muylle, E. 1988. Plasma glucose clearance and insulin response in cows with abomasal displacement. Transboundary and Emerging Diseases, 35, 221-228.

Van Winden, S. C. L., Brattinga, C. R., Muller, K. E., Schonewille, J. T. H., Noordhuizen, J. P. T. M. \& Beynen, A. C. 2004. Changes in the feed intake, $\mathrm{pH}$ and osmolality of rumen fluid, and the position of the abomasum of eight dairy cows during a diet-induced left displacement of the abomasum. Veterinary Record, 154, 501504.

Zadnik, T. 2003. Review of anterior displacement of the abomasum in cattle in Slovenia. Veterinary Record, 153, 24-25.
Zwald, N. R., Weigel, K. A., Chang, Y. M., Welper, R. D. \& Clay, J. S. 2004. Genetic selection for health traits using producerrecorded data. I. Incidence rates, heritability estimates, and sire breeding values. Journal of Dairy Science, 87, 4287-4294.

\section{Article History:}

Received 20 February 2017

Accepted 27 March 2017

Available on line 11 June 2017

License information: This is an open-access article distributed under the terms of the Creative Commons Attribution License 4.0, which permits unrestricted use, distribution, and reproduction in any medium, provided the original work is properly cited. 